\title{
Level of abstinence mediates heart-rate response to cue-elicited craving in smokers
}

\author{
Jared T. Kelly ${ }^{*} \dagger$, Sean P. Barrett ${ }^{\dagger \S}$, Robert O. Pihl ${ }^{\dagger}$, and Alain Dagher ${ }^{\S}$
}

\begin{abstract}
The subjective and autonomic components of nicotine craving were examined in abstinent and non-abstinent smokers using a cue reactivity paradigm. A cigarette abstinent condition was used to examine how smoking deprivation mediates the activity of positive and negative reinforcement mechanisms. Levels of craving and physiologic responses were monitored during the presentation of videotaped neutral and smoking stimuli. Relative to the neutral material, smoking stimuli increased several indices of subjective craving in both abstinent and non-abstinent smokers. Autonomic responses to the smoking stimuli however, appeared to be mediated by the level of abstinence. Non-deprived smokers showed an increase in heart rate during the first minute of the smoking stimuli presentation relative to the first minute of the control video, while abstinent smokers showed no change. The autonomic responses of cigarette craving may be dependent on the perceived reinforcing value of cigarettes, which can be modulated by cigarette abstinence. These findings are consistent with the hypothesis that increased heart rate may be a marker for incentive motivation, but also strongly support a multi-dimensional model of nicotine craving. Furthermore, they stress the importance of recognising the condition of the smoker as a critical determinant in craving responses.
\end{abstract}

Keywords Nicotine, craving, heart rate, abstinence, cigarette, reinforcement, autonomic, cue-reactivity

\section{INTRODUCTION}

Cravings for cigarettes have been identified as playing an important role in the continuation of smoking behaviour and in inducing smoking relapse (1). Drug-related cue exposure increases physiological and self-report measures of craving (2). In addition to smoking-related cues, cigarette deprivation also elicits craving, as the reinforcing value of cigarette smoking is increased by periods of smoking abstinence $(1,3)$. Despite the known importance of cigarette craving in the maintenance of smoking habits, the mechanisms mediating smoking urges remain uncertain.

*To whom correspondence should be addressed: Jared Kelly, 191 High St., New Glasgow, Nova Scotia, B2H 2X2 Canada email: jaredtkelly@yahoo.com

$\dagger$ Department of Psychology, McGill University

§Brain Imaging Center, Montreal Neurological Institute
Maintenance and relapse models of drug abuse have primarily been developed using a classical conditioning paradigm. The major difference in conditioning models of drug abuse is the nature of the conditioned response (CR) (2).

\section{Negative Reinforcement Models of Drug Abuse}

In Siegel's (4) model, neutral stimuli paired with drug administration can become conditioned stimuli (CS) that elicit CRs that are opposite to the physiological effects of the drug. The counterdirectional nature of the $\mathrm{CR}$ is said to compensate for the drug's pharmacological effects and help to maintain a homeostatic equilibrium. Wikler (5) and Siegel (4) both treat the CR as a negative withdrawal-like state that motivates drug relapse. Both models are also fundamentally linked to negative reinforcement mechanisms and the alleviation of conditioned withdrawal. 


\section{Positive Reinforcement Models of Drug Abuse}

The conditioned appetitive motivational model (6) proposes that the positive incentive value of drugs maintains drug-seeking behaviour. Upon experiencing a CS, an individual has a CR that is consistent with the physiological responses of the drug's positively reinforcing properties. Therefore, tobacco related CRs should elicit increased heart rate, increased sweat gland activity, and decreased skin temperature (2). A review of cue-reactivity studies supports the first two predictions, but not the predicted decrease in skin temperature (2).

\section{A Multidimensional Approach to Craving}

Although both conditioning models have received empirical support, they have also both been the subject to much criticism, as contradictory findings have limited their explanatory power (7). An alternative approach to understanding craving, proposed by Baker et al. (8), is to account for cigarette craving by incorporating positive and negative reinforcement models into one multidimensional model. In this model, two affective systems operate separately, one associated with withdrawal and negative effect, and the other with the appetitive or incentive value of drugs, and thus may offer a more complete explanation of drug addiction. Few studies have directly examined how different conditions may mediate the interaction of positive and negative reinforcement systems in cigarette craving. However, Willner et al. (1) found that the smoking behaviour of non-deprived smokers correlates with positive reinforcement, but during cigarette abstinence, smoking behaviour correlates with negative reinforcement. These findings suggest that periods of abstinence shift the control of smoking behaviour from positive reinforcement mechanisms to negative reinforcement mechanisms. Cigarette deprivation thus changes the perceived value of the cigarettes as a reinforcer (1).

\section{Study Purpose}

Several researchers object to using changes in physiological measures, such as increased heart rate and decreased skin temperature, as indicators of positively reinforced craving since physiological changes are known to be multi-determined and can provide inconsistent indices of drug-taking motivation (9). For example, heart rates of alcoholics have been found to increase or decrease in response to stimuli across different studies (13). A multidimensional view of craving may be able to address some of the inconsistencies in autonomic responses. If drug craving is a function of both positive and negative motivational systems, then it would seem plausible that autonomic responses to drug stimuli may vary with the reinforcement system in operation.

This study proposes that the condition of a smoker modulates the physiological and subjective responses to nicotine stimuli. More specifically, that a smoking abstinent condition will reduce differential heart rate responses between non-abstinent and abstinent smokers, as well as significant differences in subjective indices of craving. Such a finding would emphasize the pertinence of a multidimensional approach to drug craving studies, and the need to further investigate the various factors that modulate the relative activity of positive and negative reinforcement systems in drug users.

A smoking abstinent condition is expected to elicit nicotine's negatively reinforcing, or withdrawal, properties, while a non-abstinent condition will elicit nicotine's positively reinforcing properties. Nonabstinent smokers are thus predicted to have an increased heart rate response to nicotine cues, and abstinent smokers will have a weaker response, or even a counterdirectional response.

Dopamine levels, associated with positive reinforcement mechanisms and incentive motivation, quickly return to normal after initial stimulus presentation (10). Therefore, an increase in heart rate in non-abstinent smokers is hypothesized within the first minute of the smoking video's presentation, as the conditioned autonomic response is expected to habituate shortly after the CS introduction. The difference in heart rate responses between non-abstinent and abstinent smokers after viewing a smoking video is expected because of a partial shift from positive reinforcement to negative reinforcement due to cigarette deprivation. The proposed shift is considered partial because both mechanisms are believed to remain active, but the relative level of activity changes.

The presentation of a video replete with smoking cues is also predicted to elicit increased subjective measures of craving in smokers. Abstinent smokers are expected to have higher baseline and cue-elicited craving levels, in particular those associated with withdrawal, as well as lower positive mood scores, than non-abstinent smokers.

\section{METHODOLOGY \\ Subjects}

27 male and female undergraduates were divided into three groups: a non-abstinent smokers group $(n=9)$, an abstinent smokers group $(n=9)$, and a non-smokers group $(n=9)$. Group size was limited by constraints inherent to the study, such as time and subject pool size, but a minimum of 9 was set to ensure statistically significant results could be achieved. To be deemed a 
smoker, subjects had to smoke at least 6 cigarettes per day, every day. Non-abstinent smokers smoked immediately prior to the study, whereas abstinent smokers refrained from smoking for the previous overnight period and for a minimum of 12 hours. To be a deemed a non-smoker, subjects were required to have smoked fewer than 10 cigarettes in their lifetimes and no cigarettes within the previous two years. The inclusion criteria for each group was devised based on the guidelines followed by previous researchers and the subject population available at the time research commenced.

The experimenter was not blind to the subject grouping, as the subjects' compliance to their group guidelines needed to be verified before the experiment could begin.

\section{Materials}

Two videos were used in the study. The control video was devoid of smoking-related stimuli and merely consisted of people getting their hair cut. The experimental video contained muted images of amateur actors simulating smoking behaviour in real life settings. Such behaviour included lighting cigarettes, long and short smoke exhales, and blowing smoke rings. Both men and women were depicted in each video, and all subjects saw the same videos regardless of gender. The videos were matched for the subjective appeal of the actors to control for the confounding effect that attraction and arousal could have on physiological and subjective craving measures. Both videos were also novel to all subjects, thereby preventing the confounding effect that video or actor familiarity could have on craving indices.

A five-item questionnaire measuring subjective craving was administered while subjects viewed the videos (see Table I). The questionnaire was modeled after the Questionnaire of Smoking Urges (QSU), a 32 item questionnaire that measures subjective changes in positively and negatively reinforced craving levels in response to smoking stimuli (11). The QSU is a valid subjective measure of craving and predicts smoking behaviour (1). The QSU is also sensitive to brief periods of smoking abstinence and to smoking-related cue exposure (12). However, as subjects were required to complete the questionnaire while watching the video, a much shorter questionnaire was required. Three basic craving questions were derived from the QSU: a general cigarette craving item, a positive reinforcement associated item asking whether the subject thought a cigarette would taste good, and a withdrawal associated item concerning subjective feelings of withdrawal. Subjects drew a vertical line on a scale from 0 to 10 to answer each question.
Two additional questions were included in the questionnaire. The subject's positive mood score and the subject's level of boredom were both included, and were scored in the same manner as the craving items (see Table 1.)

Table I. Questionnaire of subjective levels of craving and mood

For each item below, draw a vertical line from 0 to 10 that

corresponds to your level of agreement with the statement.

1. I am craving a cigarette right now.

2. Smoking a cigarette would taste good right now.

3. Smoking a cigarette right now would reduce my

feelings of nicotine withdrawal.

4. I am in a positive mood right now.

5. I am bored right now.

\section{Procedure}

Institutional ethics approval was obtained before the onset of the study. Upon arrival to the study room and before beginning the experiment, all subjects signed an informed consent form.

After meeting in a cigarette cue-free study room, all subjects verbally confirmed their smoking status and had their $\mathrm{CO}$ levels measured to verify compliance. A maximum $10 \mathrm{CO}$ ppm cutoff was used for abstinent smokers, and 0 ppm for non-smokers. All three groups had baseline heart rate measurements taken, followed by completion of the subjective questionnaire, before viewing either video.

The control video was presented first for all subjects, in order to prevent a potential confound of residual craving effects, and was immediately followed by the experimental video. While heart rate was recorded continuously throughout both videos, the craving questionnaire was given after 30 seconds and 4.5 minutes into each video. The 30 second time slot was selected in order to capture any initial and/or transitory changes in subjective craving, and to temporally correspond with the hypothesized changes in heart rate.

\section{Statistical Analyses}

An alpha level of 0.05 was used for all statistical analyses. Subjective cravings, mood, boredom, and heart rate data were initially analysed by a repeatedmeasures analysis of variance (ANOVA) for the overall sample. A Tukey HSD post hoc test was used to analyse craving differences in subjects at baseline. Paired samples t-tests were used to analyse changes in the smokers' subjective scores of craving, mood, and boredom, across baseline and the two video conditions. A different statistical procedure was employed when the smokers group was further divided into abstinent and non-abstinent groups for analysis. Due to the small 
number of subjects in each group, as well as missing data in some cells, non-parametric Wilcoxon Signed Ranks tests were used to analyse abstinent and nonabstinent smokers' changes in subjective scores of craving, mood, boredom, $\mathrm{CO}$ levels, and changes in heart rate, across baseline and the two video conditions. Mann-Whittney $U$ tests were performed to analyze heart rate and craving differences between smoking groups at baseline.

\section{RESULTS}

\section{Subjects}

Subjects were all between 17 and 23 years of age. Abstinent smokers had significantly lower CO levels prior to testing $(\mathrm{M}=4.80, \mathrm{SD}=1.9235)$ than did nonabstinent smokers $(\mathrm{M}=10.0, \mathrm{SD}=4.06 ; \mathrm{Z}=2.2, \mathrm{p}=$ .03.) Non-abstinent smokers and abstinent smokers did not vary significantly in daily cigarette consumption (non-abstinent $\mathrm{M}=12.39$; abstinent $\mathrm{M}=10.50$ ), nor did they vary in number of years smoking (non-abstinent $\mathrm{M}$ = 4.56; abstinent $\mathrm{M}=3.89$ respectively).

\section{Non-smokers}

Non-smokers did not experience subjective craving at baseline or at any point during the video presentations. The heart rate of non-smokers also did not significantly change at any point during the experiment.

Subjective responses to video presentation

No differences in subjective craving, including positively and negatively reinforced craving, were found between baseline levels and after 30 seconds of the control video across either of the smoking conditions.

Subjective cigarette craving across all smokers increased significantly after 30 seconds of the smoking video presentation ( $\mathrm{M}=5.76, \mathrm{SD}=2.80)$ compared to baseline craving levels $(\mathrm{M}=4.36, \mathrm{SD}=3.11 ; \mathrm{t}(17)=$ $5.225, \mathrm{p}<0.001)$. All items of subjective cigarette craving were increased in smokers, after 30s and over the entire smoking video, relative to the control video (see Table II.)

\section{Effects of abstinence on baseline subjective craving}

Baseline measurements of subjective craving revealed that cigarette abstinence modulates nicotine craving. Abstinent smokers had greater levels of general craving $(\mathrm{M}=5.85, \mathrm{SD}=2.92)$ than nonabstinent smokers at baseline $(\mathrm{M}=2.86, \mathrm{SD}=2.65 ; \mathrm{p}=$ 0.0274.) The positive $(\mathrm{M}=7.37, \mathrm{SD}=2.22)$ and the negative $(\mathrm{M}=6.64, \mathrm{SD}=2.66)$ reinforcement items of craving in abstinent smokers were also elevated relative to non-abstinent smokers' positive $(\mathrm{M}=3.89, \mathrm{SD}=$ $2.97 ; \mathrm{p}=0.006)$ and negative $(\mathrm{M}=2.85, \mathrm{SD}=2.18 ; \mathrm{p}$ $=0.001)$ reinforcement items.
Table II. Smokers' subjective responses to smoking video

\begin{tabular}{|c|c|c|c|c|}
\hline \multicolumn{5}{|c|}{ Video condition - after $30 \mathrm{~s}$} \\
\hline Cravins index & Control & Smoking & $\mathrm{T} 1$ & $\mathrm{~T} 2$ \\
\hline general & $4.66(3.10)$ & $5.76(2.80)$ & 5.225 & $3.727 *$ \\
\hline tastes good & $5.04(2.62)$ & $6.29(2.62$ & & $3.510^{*}$ \\
\hline withdrawl & $4.52(2.87)$ & $5.63(2.92)$ & & $3.442 *$ \\
\hline \multicolumn{5}{|l|}{$*$ prob $<0.01$} \\
\hline \multicolumn{5}{|c|}{ Video condition - entire video } \\
\hline Cravins index & Control & Smoking & $\mathrm{T} 1$ & $\mathrm{~T} 2$ \\
\hline general & $4.70(2.82)$ & $5.69(2.73)$ & & $4.313 * *$ \\
\hline tastes good & $5.30(2.47)$ & $6.26(2.54)$ & & $4.311 * *$ \\
\hline withdrawl & $4.67(2.76)$ & $5.75(2.80)$ & & $4.112^{* *}$ \\
\hline
\end{tabular}

$* *$ prob $<0.01$

\section{Effects of abstinence on cue-associated subjective craving}

Although smokers as a whole experienced increased subjective levels of positively and negatively reinforced craving upon smoking video presentation, analysis of non-abstinent and abstinent smokers as two separate groups yielded different results.

Each of the non-abstinent smokers' craving indices were elevated in the smoking video relative to the control video (see Table III). Interestingly, the withdrawal item was not significant after 30 s but was for the video as a whole, suggesting withdrawal increased throughout video presentation.

In abstinent smokers, presentation of the smoking video significantly increased both general craving $(\mathrm{p}<0.05)$ and incentive motivation $(\mathrm{p}<0.05)$ craving relative to the control video. There were no significant differences between the conditions for withdrawal motivated craving (see Table III).

\section{Positive mood and boredom}

Cigarette abstinence did not affect mood in smokers, as abstinent and non-abstinent smokers did not vary significantly in positive mood scores. Non-smokers mood scores were also statistically equivalent to both groups of smokers. Positive mood and boredom scores did not differ significantly among or between the three groups from baseline through the control and experimental conditions. 
Table III. Subjective craving ratings for non-abstinent smokers

\begin{tabular}{|c|c|c|c|c|c|}
\hline Craving type & Time period & Video & $\mathbf{M}$ & SD & $\mathbf{Z}$ \\
\hline General & $\begin{array}{l}\text { Entire video } \\
\text { smoking }\end{array}$ & $\begin{array}{l}\text { control } \\
4.43\end{array}$ & $\begin{array}{l}3.47 \\
2.62\end{array}$ & 2.67 & $2.53^{*}$ \\
\hline Tastes good & $\begin{array}{l}\text { Entire video } \\
\text { smoking }\end{array}$ & $\begin{array}{l}\text { control } \\
5.06\end{array}$ & $\begin{array}{l}4.16 \\
2.66\end{array}$ & 2.69 & $2.55^{*}$ \\
\hline Withdrawal & $\begin{array}{l}\text { Entire video } \\
\text { smoking }\end{array}$ & $\begin{array}{l}\text { control } \\
4.08\end{array}$ & $\begin{array}{l}3.01 \\
2.56\end{array}$ & 2.43 & $2.67 * *$ \\
\hline General & After $30 \mathrm{~s}$ & $\begin{array}{l}\text { control } \\
\text { smoking }\end{array}$ & $\begin{array}{l}3.37 \\
4.19\end{array}$ & $\begin{array}{l}2.74 \\
2.54\end{array}$ & $2.56^{*}$ \\
\hline Tastes good & After $30 \mathrm{~s}$ & $\begin{array}{l}\text { control } \\
\text { smoking }\end{array}$ & $\begin{array}{l}4.11 \\
4.87\end{array}$ & $\begin{array}{l}2.67 \\
2.66\end{array}$ & $2.57 *$ \\
\hline Withdrawal & After $30 \mathrm{~s}$ & $\begin{array}{l}\text { control } \\
\text { smoking }\end{array}$ & $\begin{array}{l}6.16 \\
7.41\end{array}$ & $\begin{array}{l}2.51 \\
2.14\end{array}$ & $1.91^{\mathrm{tt}}$ \\
\hline
\end{tabular}

$\mathrm{t}$ prob $=0.063, \mathrm{tt}$ prob $=0.056, *$ prob $<0.05$

Table IV. Heart rate averages of non-smokers and smokers during video presentations

\begin{tabular}{|c|c|c|c|c|c|}
\hline Smoker type & Time period & Video & $\mathbf{M}$ & SD & $\mathbf{Z}$ \\
\hline General & Entire video & $\begin{array}{l}\text { control } \\
\text { smoking }\end{array}$ & $\begin{array}{l}5.94 \\
6.95\end{array}$ & $\begin{array}{l}2.52 \\
2.33\end{array}$ & $2.20 *$ \\
\hline Tastes good & Entire video & $\begin{array}{l}\text { control } \\
\text { smoking }\end{array}$ & $\begin{array}{l}6.44 \\
7.45\end{array}$ & $\begin{array}{l}1.66 \\
1.86\end{array}$ & $2.08 *$ \\
\hline Withdrawal & Entire video & $\begin{array}{l}\text { control } \\
\text { smoking }\end{array}$ & $\begin{array}{l}6.33 \\
7.41\end{array}$ & $\begin{array}{l}2.01 \\
1.96\end{array}$ & $1.79^{t}$ \\
\hline General & After $30 \mathrm{~s}$ & $\begin{array}{l}\text { control } \\
\text { smoking }\end{array}$ & $\begin{array}{l}5.96 \\
7.32\end{array}$ & $\begin{array}{l}3.03 \\
2.18\end{array}$ & $2.19 *$ \\
\hline Tastes good & After $30 \mathrm{~s}$ & $\begin{array}{l}\text { control } \\
\text { smoking }\end{array}$ & $\begin{array}{l}5.97 \\
7.72\end{array}$ & $\begin{array}{l}2.35 \\
1.74\end{array}$ & $2.21 *$ \\
\hline Withdrawal & After $30 \mathrm{~s}$ & $\begin{array}{l}\text { control } \\
\text { smoking }\end{array}$ & $\begin{array}{l}6.16 \\
7.41\end{array}$ & $\begin{array}{l}2.51 \\
2.14\end{array}$ & $1.91^{\mathrm{tt}}$ \\
\hline
\end{tabular}

$\mathrm{t}$ prob $=0.063, \mathrm{tt}$ prob $=0.056, *$ prob $<0.05$

\section{Physiological responses to video presentation}

At baseline, a Mann-Whitney test revealed a trend toward an elevated heart rate in non-abstinent smokers $(\mathrm{M}=84.63, \mathrm{SD}=19.66)$ relative to abstinent smokers $(\mathrm{M}=66.92, \mathrm{SD}=6.78 ; \mathrm{Z}=-1.85, \mathrm{p}=.06)$.

Presentation of the smoking video did not elevate the heart rate of the smokers group as a whole relative to the control video condition (See Table IV for the heart rates of smokers and non-smokers during the video presentations). However, when non-abstinent and abstinent smokers were analyzed separately using
Wilcoxon Signed Ranks Tests, the non-abstinent smokers had a significantly elevated heart rate during the first minute of the smoking video compared to during the first minute of the control video $(Z=-2.5$, $p$ $=.013)$. The abstinent smokers' heart rate did not increase during the first minute of the smoking video compared to the first minute of the control video $(\mathrm{Z}=$ $0.2, \mathrm{p}=.916)$.

Due to equipment malfunction, the heart rate data was incomplete for 4 subjects (3 abstinent, 1 non-smoker.) It was thus possible that our failure to detect an effect of the smoking video on heart rate in the abstinent condition was due to a lack of statistical power for this condition. In order to minimize this possibility, a chisquared test was preformed to compare the proportion of subjects evidencing an increase in heart rate during the smoking video in the abstinent and non-abstinent conditions. Analyses revealed a significantly greater proportion of non-abstinent smokers (88.9\%) experiencing an increase in heart rate relative to abstinent smokers $(50 \%$; chi-squared $=2.74, \mathrm{p}=.047$ [one tailed]).

\section{DISCUSSION}

This study was intended to investigate the effect cigarette abstinence plays in subjective and autonomic responses to nicotine stimuli. Furthermore, it was postulated that an abstinent condition would result in lower heart rates and higher levels of subjective craving compared to a non-abstinent smokers' group. The obtained results support this hypothesis and in doing so, provide empirical support for a multidimensional model of craving; a model that stipulates that the condition of the drug user can modulate the relative activity of positive and negative reinforcement systems. This model stresses the importance of recognising the condition of the drug user when conducting craving studies.

With respect to subjective craving, the smoking video was found to elicit all three craving indices in smokers: general craving, tastes good, and withdrawal, and have no effect on non-smokers. Although only one item was used to measure positively and negatively reinforced craving, the results support the notion that both mechanisms may be active in cue-elicited nicotine craving. This evidence supports a multidimensional model of cue-associated cigarette craving.

In both the abstinent and non-abstinent groups, the overwhelming trend was an increase in all three subjective items of craving in response to the smoking video. Surprisingly, the cue-elicited responses of abstinent smokers were elevated for the first two craving items (general craving and tastes good) but not for subjective levels of withdrawal. However, this 
finding may be partly due to the extreme wording of the withdrawal item, relative to the tastes good item. Also, abstinent scores may have been limited by a ceiling effect, as subjective craving at baseline was elevated in abstinent smokers compared to non-abstinent smokers across all three craving items, thereby providing less potential 'room' for elevated scores.

This latter finding, that baseline craving scores are higher for abstinent smokers, concurs with previous research (12) and further stresses the importance of recognizing the condition of the smoker. This fact alone, that smokers can begin a study with different levels of craving, is sufficient reason to take abstinence into consideration when conducting craving studies.

This argument is further supported with the complementary results of subjective and autonomic craving indices. Non-abstinent smokers had a strong statistical trend towards having an elevated heart rate relative to abstinent smokers at baseline. This result is consistent with previous findings that nicotine administration increases heart rate and nicotine withdrawal decreases heart rate (13). This physiological evidence complements the subjective baseline evidence, revealing that abstinent and non-abstinent smokers began the study both in a different subjective state and with different physiology.

With regards to a multidimensional model of cueelicited craving, the strongest support in this study comes from the heart rate responses in abstinent and non-abstinent smokers. A quick overview of positive and negative reinforcement models helps provide clarity to this conclusion.

Withdrawal based conditioning models predict a conditioned response (CR) for heart rate that is counterdirectional to the effects of the drug; thus they predict no increase or a decrease in heart rate in response to the smoking video. Conversely, appetitive or positive reinforcement conditioning models predict an isodirectional $\mathrm{CR}$ to a smoking conditioned stimulus (CS). Therefore, these models predict an increase in heart rate in response to this study's smoking video.

This study's findings thus reveal that both models' predictions have merit. Non-abstinent smokers had an increase in heart rate after one minute of the smoking video, while abstinent smokers had no increase. These differences in autonomic responses support Willner's (1) conclusion that smoking abstinence shifts nicotine craving from positive to negative reinforcement mechanisms. The autonomic responses of cigarette craving may thus be dependent on the perceived reinforcing value of cigarettes, which can be modulated by cigarette abstinence. We propose that the condition of the smoker, such as abstinent or non-abstinent, modulates the relative activity of positive and negative
Table V. Heart rate averages of non-smokers and smokers during video presentations

\begin{tabular}{lllllc}
\hline Smoker type & Time period & Video & M & N & SD \\
\hline Non-smokers & Entire video & control & 72.04 & 8 & 8.17 \\
& & smoking & 72.18 & 8 & 9.00 \\
Non-smokers & First minute & control & 72.75 & 8 & 7.07 \\
Non-smokers & First minute & smoking & 73.88 & 8 & 7.42 \\
& & & & & \\
All smokers & Entire video & control & 77.06 & 15 & 16.89 \\
All smokers & Entire video & smoking & 78.40 & 15 & 16.1 \\
& & & & & \\
All smokers & First minute & control & 78.73 & 15 & 17.61 \\
All smokers & First minute & smoking & 81.07 & 15 & 16.57 \\
& & & & & \\
Non-abstinent & Entire video & control & 82.93 & 9 & 19.5 \\
Non-abstinent & Entire video & smoking & 84.27 & 9 & 18.49 \\
& & & & & \\
Non-abstinent & First minute & control & 81.67 & 9 & 20.94 \\
Non-abstinent & First minute & smoking & 85.56 & 9 & 19.84 \\
& & & & & \\
Abstinent & Entire video & control & 68.25 & 6 & 5.98 \\
Abstinent & Entire video & smoking & 69.59 & 6 & 4.88 \\
& & & & & \\
Abstinent & First minute & control & 74.33 & 6 & 11.33 \\
Abstinent & First minute & smoking & 74.33 & 6 & 6.95 \\
\hline
\end{tabular}

reinforcement mechanisms, and thereby mediates the conditioned autonomic responses to smoking stimuli.

The fact that heart rate was significantly increased after one minute, but not over the entire video, is not surprising considering dopamine habituation studies. Cue-elicited dopamine release quickly returns to normal after initial stimulus presentation (10). As dopamine release has been linked to increases in heart rate, its habituation after CS presentation thus coincides with our finding of increased heart rate in non-abstinent smokers only within the first minute of stimulus presentation.

\section{Study implications and limitations}

The results obtained in this study offer promising insight into understanding the physiological mechanisms of nicotine craving. They also emphasize the critical importance of examining drug craving as a dynamic entity that occurs across variable contexts. Clearly, the study's key empirical finding is that cigarette abstinence modulates cue-elicited heart rate responses. This finding strongly supports a multidimensional view of craving, one that encompasses both the positive and negative reinforcing properties of drug use.

It may also help to clarify some of the seemingly contradictory findings of other studies of cue-elicited autonomic responses $(2,9)$. For if the nature of conditioned responses are investigated with due consideration given to the multiple variables that 
influence a subject's psychological and physiological state, such as drug abstinence or perceived availability, then consistent results may be more readily obtained. Such results would be a more valid reflection of the underlying mechanisms of drug craving. In this study, dividing smokers along the lines of a smoking abstinent condition resulted in two distinct heart rate responses. These results, coupled with heightened levels of subjective craving in abstinent smokers, provide strong impetus to conduct research that regards craving as a complex phenomenon that is influenced by many situational and physiological factors.

With respect to situational factors, one in particular necessitates further research: perceived drug availability. Subjects in this study ostensibly knew they could smoke immediately after its completion. This awareness may have influenced subjective and autonomic responses to nicotine stimuli. For example, in abstinent smokers, the perceived availability of cigarettes may heighten appetitive craving responses across subjective and autonomic indices, whereas a perceived unavailable condition could increase withdrawal motivated craving responses. Furthermore, a perceived unavailable condition warrants future study if for no other reason than because it more closely resembles real-life attempts at quitting smoking.

This study's heart rate results warrant additional research into the neural basis of nicotine craving. Changes in heart rate intuitively suggest corresponding changes in neural activity. In particular, a positive correlation between heart rate responses and dopamine release could be hypothesized, as dopamine release is associated with increases in heart rate. Thus, an fMRI study could reveal that the differences in heart rate responses between abstinent and non-abstinent smokers correlates to differences in dopamine release. Any such study should also give attention to baseline neural activity. As abstinent smokers were observed to have lower heart rates and reported higher levels of subjective craving that non-abstinent smokers at baseline, it seems plausible that neural imaging could reveal differential baseline neural activity between the two smoking groups. That one can only speculate such results is sufficient evidence of the paucity of available literature on the neural correlates of nicotine craving, and is motivation for future study.

In this study, there are a couple of limitations that merit discussion and provide opportunities for additional research. Perhaps most limiting is the number of subjective and autonomic measures of craving. Because of the pragmatics of the video presentation, craving items were restricted to one item each for general craving, positive reinforcement, and negative reinforcement. A more extensive questionnaire, such as the QSU, would have been more sensitive to different types of craving and may have offered more insight into possible links between subjective and autonomic craving indices. Additional physiological measures of craving, such as skin conductance and temperature, and neural imagery, could provide a more complete view of how smoking stimuli mediate conditioned autonomic responses. A second limitation to the study is not altering the sequence of the control and smoking videos, in order to eliminate the possibility of residual craving effects from the smoking video. However, a comparison of subjective and autonomic craving measurements between baseline and the control video condition, a 20 minute time difference, yielded no differences. Therefore, it is unlikely that the increase in smokers' craving in the experimental video is due to the four minute lag between the control and experimental video presentations. Lastly, it would be valuable to replicate the heart rate data with a larger sample size, thereby confirming our current understanding of the effects of abstinence on physiological responses to craving stimuli.

It is imperative that future research examine cueelicited craving in a manner that is mindful of the myriad number of factors that may influence subjective and physiological indices of craving. If the mechanisms of drug craving are to be fully understood, the condition of drug users must be recognized as a critical determinant in their responses to drug stimuli.

\section{ACKNOWLEDGEMENTS}

Thanks to Mark Gross for creating the software responsible for analyzing subjects' heart rate responses.

\section{REFERENCES}

1. Willner, P., Hardman, S., \& Eaton, G. Subjective and behavioural evaluation of cigarette cravings. Psychopharmacology, 118: 171-177; 1995.

2. Carter, B.L., \& Tiffany, S.T. Meta-analysis of cue-reactivity in addiction research. Addiction, 94(3): 327-340; 1999.

3. Perkins, K.A., Epstein, L.H., Grobe, J., \& Fonte, C. Tobacco abstinence, smoking cues, and the reinforcing value of smoking. Pharamacology Biochemistry and Behaviour, 47: 107-112; 1994.

4. Siegel, S. Classical conditioning, drug tolerance, and drug dependence. In R.G. Smart, F.B. Glaser, Y. Israel, et al. (Eds.), Research advances in alcohol and drug problems, 7: 207-246. New York: Plenum, 1983.

5. Wikler, A. Recent progress in research on the neurophysiological basis of morphine addiction. American Journal of Psychiatry, 105: 329-338; 1948.

6. Stewart, J., De Wit, H., \& Eikelboom, R. The role of unconditioned and conditioned drug effects in the selfadministration of opiates and stimulants. Psychological Review, 91: 251-268; 1984.

7. Robinson, T.E., \& Berridge, K.C. The neural basis of drug craving: an incentive-sensitization theory of addiction. Brain Research Reviews, 18: 247-291; 1993. 
8. Baker, T.B., Morse, E., \& Sherman, J.E. The motivation to use drugs: psychobiological analysis of urges, in: C. Rivers (Ed.). The Nebraska Symposium on Motivation: alcohol use and abuse: 257-323. Lincoln, Nebraska: University of Nebraska Press, 1987.

9. Piasecki, T.M., Smith, S.S., Baker, T.B. (1999). The tyranny of habit and availability. From: Comments on Carter \& Tiffany's "Meta-analysis of cue-reactivity in addiction research". Addiction, 94 (3): 341-351.

10. Schultz, W. Predictive reward signal of dopamine neurons. Journal of Neurophysiology, 80: 1-27; 1998.
11. Tiffany, S.T., \& Drobes, D.J. (1991). The development and initial validation of a questionnaire on smoking urges. British Journal of Addiction, 86: 1467-1476.

12. Morgan, M.J., Davies, G.M., \& Willner, P. The Questionnaire of Smoking Urges is sensitive to abstinence and exposure to smoking-related cues. Behavioural Pharmacology, 10, No 6/7 619-626: 1999.

13. Niaura, R.S., Rohsenow, D.J., Binkoff, J.A., Monti, P.M., Pedraza, M., \& Abrams, D.B. Relevance of cue reactivity to understanding alcohol and smoking relapse. Journal of Abnormal Psychology, 97, 133-152: 1988.

Jared Kelly obtained a B.Sc. in Honours Psychology from McGill University. He has conducted research in the Department of Psychology at McGill University, under the supervision of Alain Dagher, from the Montreal Neurological Institute. His current interests include health care in the developing world, with emphasis on conflict/crisis situations and international criminal tribunals. 